\title{
GLOBAL SOLUTIONS OF NEUTRINO DOMINATED ACCRETION FLOWS AROUND KERR BLACK HOLES
}

\author{
LI XUE*, TONG LIU, WEI-MIN GU and JU-FU LU \\ Department of Physics and Institute of Theoretical Physics and Astrophysics \\ Xiamen University, Xiamen, Fujian 361005, China \\ *lixue@xmu.edu.cn
}

\begin{abstract}
By solving a set of coupled hydrodynamical and microphysical equations with some appropriate boundary conditions, we obtain three global solutions of typical neutrinodominated accretion flows around different spinning black holes. Our results reveal that the effect of black hole spin on the flows is restricted within inner parts of neutrinodominated regions.
\end{abstract}

Keywords: Accretion; accretion disks; black hole physics; gamma rays bursts.

\section{Introduction}

Theoretical models for gamma-ray bursts (GRBs) can be divided into two categories: the fireball models, which treat the shock interaction of relativistic outflows and the production of gamma rays and afterglows in other wavelengths ${ }^{1-2}$, and those that explore the central engine of the fireball, that is, the energy source for the relativistic outflows. The most popular models in the latter category have in common the invocation of a stellar mass black hole accreting at a hypercritical rate, on the order of $1 M_{\odot} \mathrm{s}^{-1}$. The main problem in these models is how to convert some fraction of the gravitational energy released by the accreted matter into a relativistic outflow to create an explosion with energy $\sim 10^{50}-10^{52}$ ergs (depending on whether the emission is isotropic or beamed). Two mechanisms have been proposed to tackle this problem: neutrino emission and annihilation, and energy extraction from the accretion disk or black hole by means of magnetohydrodynamic processes $^{3-4}$. The former, namely the neutrino dominated accretion flow (NDAF), is easier to understand and can be calculated more accurately, so it is the topic of this paper.

\section{Model}

Our model is composed of the hydrodynamical equations and the microphysical equations of hot and dense nuclear matter. The hydrodynamical equations include the continuity equation, gas energy equation, radial momentum equation, angular 

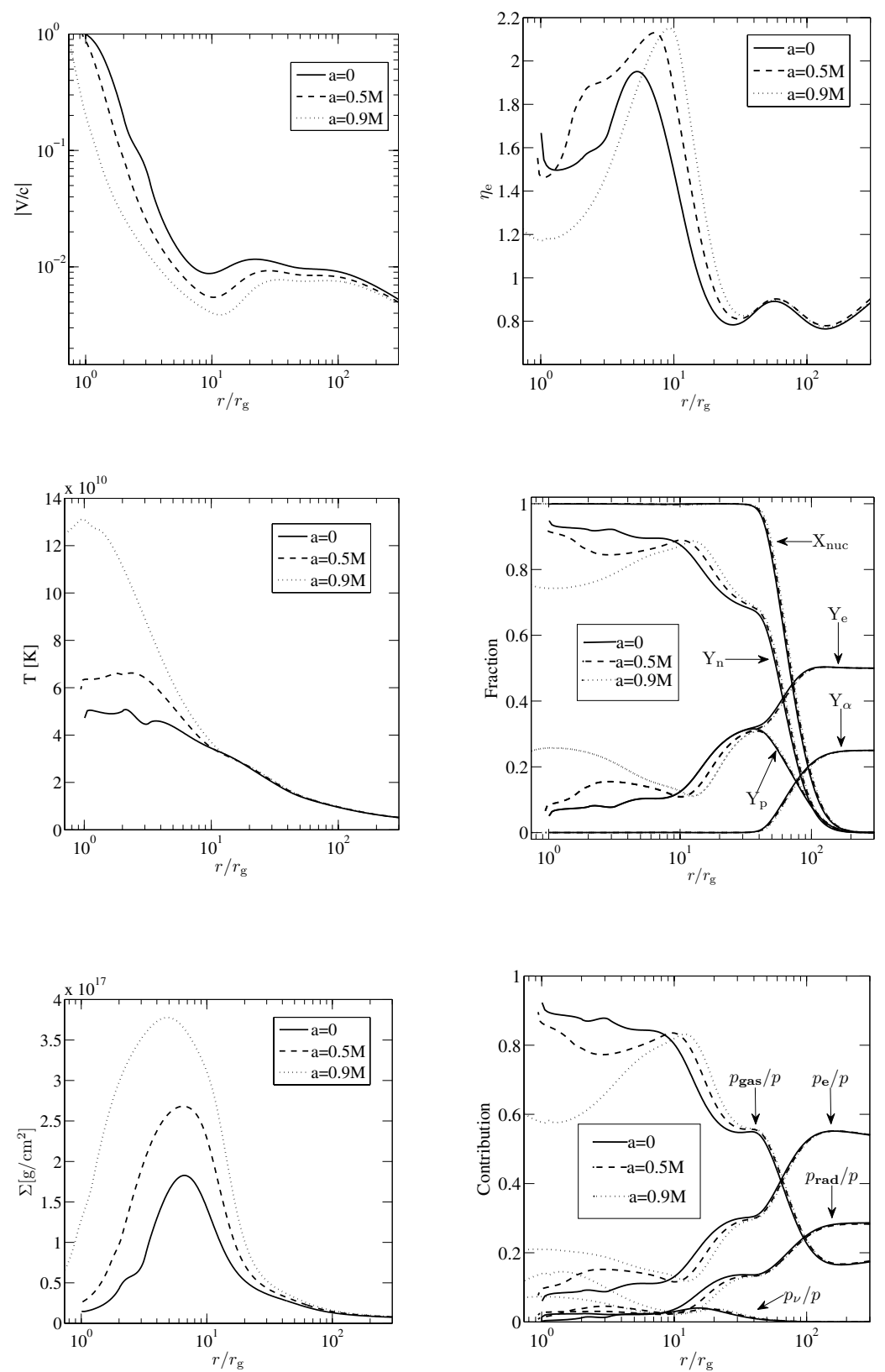

Fig. 1. The profiles from three solutions with the same viscosity parameter $\alpha=0.1$ and accretion rate $\dot{M}=1 M_{\odot} / \mathrm{s}$, but with different black hole spins. In the left column, the plots are for the radial velocity, temperature and surface density from top to bottom respectively. The top-right plot is for the electron degeneracy, middle-right plot is for the particle fractions $\left(\mathrm{Y}_{\mathrm{e}}, \mathrm{Y}_{\mathrm{p}}, \mathrm{Y}_{\mathrm{n}}, \mathrm{Y}_{\alpha}\right.$, and $\mathrm{X}_{\text {nuc }}$ denote the fractions of electron, proton, neutron, $\alpha$ particle, and free nucleons respectively), and the bottom-right plot is for the pressure contributions ( $p_{\text {gas }}, p_{\mathrm{rad}}, p_{\mathrm{e}}$, and $p_{\nu}$ are the gas, radiation, electron, and neutrino pressures respectively). 
momentum conservation equation, and the equation of vertical mechanical equilibrium. We follow Refs. 3 and 5 to derive those equations in Kerr metric, but we only follow Ref. 5 to implement the standard $\alpha p$ viscosity instead of the causal one in Ref. 3 in order to reduce the difficulty for solving those equations. In practice, the effect of different viscosities on solutions is very little.

In order to close the system, we need to define the total pressure and total cooling rate, which appear in the hydrodynamical equations. We follow our previous work Ref. 6 to consider the accreted gas composed of $\alpha$-particles and free nucleons. Therefore, the total pressure is contributed by the gas, photon, pair of electron and positron, and neutrino, and the total cooling rate is comprised of photon, neutrino, and photodisintegration of $\alpha$-particle cooling rates (see Ref. 6 for the detailed microphysical equations).

\section{Conclusions}

By solving those coupled hydrodynamical and microphysical equations with some appropriate boundary conditions, we can obtain the global solution of accretion disk structure. In Fig. 1, we show the profiles according to the solutions of three typical NDAFs around different spinning black holes $(a=0,0.5 M, 0.9 M$, in geometrical units, $M$ is black hole mass). There are six plots in this figure. The three ones in left column reveal the hydrodynamical features, and the other three ones in right column are for the relevant microphysical distributions. All of those profiles reveal that the effect of black hole spin on the structure of NDAFs is restricted in the regions from event horizon to about $40 r_{g}\left(r_{g} \equiv 2 M\right.$, gravitational radius), meanwhile the neutrino-dominated regions are all within about $100 r_{g}$.

\section{Acknowledgments}

This work was supported by 973 Program under grant 2009CB824800; the National Natural Science Foundation of China under grants 10833002, 11003016, 11073015, and 11103015; and the Natural Science Foundation of Fujian Province of China under grant 2010J01017.

\section{References}

1. P. Mészáros, Annual Rev. of Astron. and Astrophy. 40 (2002) 137.

2. B. Zhang and P. Mészáros, Int. J. Mod. Phys. A 19 (2004) 2385.

3. R. Popham, S. E. Woosley, and C. Fryer, Astropy. J. 518 (1999) 356.

4. T. Di Matteo, R. Perna, and R. Narayan, Astropy. J. 579 (2002) 706.

5. A. Sądowski, Astropy. J. Suppl. Series 183 (2009) 171.

6. T. Liu, W.-M. Gu, L. Xue and J.-F. Lu, Astropy. J. 661 (2007) 1025. 